\title{
Half-Cycle-Waveform-Inversed Single-Carrier Seven-level Sinusoidal Modulation
}

\author{
Fengjiang $\mathrm{Wu}^{\dagger}$, Bo Sun ${ }^{*}$, Lujie Zhang ${ }^{*}$, and Li Sun ${ }^{*}$ \\ ${ }^{\dagger *}$ Dept. of Electrical Engineering, Harbin Institute of Technology, Harbin, China
}

\begin{abstract}
A half-cycle-waveform inversion based three reference modulations seven-level SPWM (TRM-SPWM) scheme with one carrier is proposed in this paper. To keep the same comparison logics for the modulations and carrier during the negative half cycle and the positive one for the modulations, in the negative half cycle of the modulations, the DC offsets related to the amplitude of the carrier are set on the three modulations, respectively. The seven-level SPWM waveform with dead time thereby is implemented with only one Digital Signal Processor (DSP) without any other attached logic circuit. The basis principle of the proposed TRM-SPWM is analyzed in detail, and the frequency spectrums of the conventional and the proposed schemes are derived and compared with each other through simulation. The DSP based implementation is presented and detailed experimental waveforms verify the accuracy and feasibility of the proposed TRM-SPWM scheme.
\end{abstract}

Key words: Half cycle waveform inversion, Seven-level sinusoidal pulse width modulation, Three modulations

\section{INTRODUCTION}

In recent years, multilevel inverters have become more attractive due to their advantages over two-level inverters, such as lower electromagnetic interference, lower acoustic noise, low core losses, smaller filter size, and so on [1]-[4].

Various topologies for multilevel inverters have been proposed over the years. Common topologies include the diode-clamped [5], flying capacitor [6] and cascaded H-bridge inverters [7]. In a single phase system, the diode-clamped inverter has been widely applied due to the fact that it has fewer switching components than other topologies. Accordingly, multi-carrier based multilevel SPWM strategies suitable for diode-clamped inverters have been presented [8], [9].

Nowadays, DSPs are applied widely to implement two-level PWM algorithms, but it is difficult to implement the multi-carrier based multilevel SPWM [10]. In [11], [12], a three-level SPWM is implemented with a DSP+CPLD platform. The synchronization error between the two timers in the DSP for generating two carriers results in distortions in the output voltage waveform. In [13], the DSP+FPGA platform is used. This scheme reduces the burden of the DSP, but the cost

Manuscript received Jul. 24, 2012; revised Nov. 5, 2012

Recommended for publication by Associate Editor Sanjeet K. Dwivedi.

*Corresponding Author: shimeng@hit.edu.cn

Tel: +86-451-86412946, Fax: +86-451-86412946, Harbin Inst. of Tech.

*Dept. of Electrical Engineering, Harbin Institute of Technology, China of the FPGA is high and the development period is long. The separate implementation schemes have lower reliability and the resources of the DSP are not fully utilized.

A single carrier three-level SPWM scheme has been proposed by Salam et al. [14]. Similarly, a three-modulation and one carrier based seven-level SPWM scheme has been presented in [15]. However, the SPWM schemes in [14] and [15] used the same waveforms in the positive and negative half cycles of the modulations. In the negative half cycle of the reference modulation, the comparison logics between the three half cycles and the carrier need to be inversed to implement multilevel output PWM waveforms. The DSP can only implement the same logic comparison in the positive half cycle and the negative one. As a result, the attached digital logic circuits and the dead time generation circuit are still needed to switch the control signals of various power switches in the negative half cycles of the three modulations.

On the basis of the idea of single carrier and multi modulations of multilevel SPWM, this paper proposes a novel TRM-SPWM, which is more convenient to implement with a DSP and other attached digital logic chips are not needed. The system is more compact, the cost is reduced and the reliability is increased.

\section{PRINCIPLE OF TRM-SPWM}

A schematic diagram of a single-phase seven-level asymmetry diode-clamped inverter is shown in Fig. 1. The left 


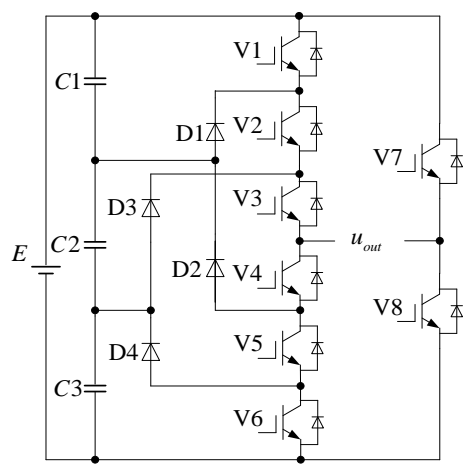

Fig. 1. Schematic diagram of eight switches seven-level inverter.

bridge arm is combined with a five-level diode-clamped leg and the right bridge arm is combined with a two-level leg. The dead time should be set so that it is between pairs of complementary switches, V1 and V4, V2 and V5, V3 and V6, and V7 and V8.

The principle behind the conventional single-carrier multimodulation based multilevel SPWM is analyzed as follows. A seven-level SPWM waveform is shown in Fig.2. Three modulations are compared with one carrier to implement the seven level output voltage waveform. It can be seen from Fig.2 that the comparison results of the modulations and the carrier in positive half cycle can be used as the control signals of various power switches to generate a multilevel voltage waveform. However, in negative half cycle, the comparison results can not be used to control the various power switches. Therefore, the comparison results are reconfigured. The logics between the actual control signals (GV1-GV6) and the comparison results (S1-S6) are:

$$
\left\{\begin{array}{l}
G_{V 1}=S 1 \& S 4+\overline{S 3} \& \overline{S 4} \\
G_{V 2}=S 2 \& S 4+\overline{S 2} \& \overline{S 4} \\
G_{V 3}=S 3 \& S 4+\overline{S 1} \& \overline{S 4} \\
G_{V 4}=\overline{S 1} \& S 4+S 3 \& \overline{S 4} \\
G_{V 5}=\overline{S 2} \& S 4+S 2 \& \overline{S 4} \\
G_{V 6}=\overline{S 3} \& S 4+S 1 \& \overline{S 4}
\end{array}\right.
$$

It can be seen that there are two problems in this scheme. The first one is that it can not be implemented directly by the PWM generation module in a DSP. The second one is due to the fact that the control signal of any component in the left bridge arm can not be obtained simply from inversing other control signals, which means that the dead time generation module in a DSP can not be used. Thus the attached logic chip and dead time generation circuit should be added to implement the PWM scheme. As a result, the system is complicated and the reliability is reduced.

To solve the above problems, a novel TRM-SPWM scheme is proposed, and the principle is shown in Fig. 3. The three modulations are compared with the same carrier. To obtain the seven-level SPWM waveform without inverting the comparison logic, the three modulations in their negative half cycles are all inverted and offset with different DC values (related to the amplitude of the carrier). The comparison result of $u_{r 1}$ and the carrier is used as the control signal of V1, where logic inversion is used as the control signal of V4. The comparison result of $u_{r 2}$ and the carrier is used as the control signal of V2, and its logic inversion is employed as the control signal of V5. The comparison result of $u_{r 3}$ and the carrier is utilized as the control signal of V3, and its logic inversion is used as the control signal of V6. In the positive half cycle, V8 is on, and V7 is off. In the negative half cycle, V8 is off and V7 is on.

The functions of the three modulations are derived as follows. Assuming that the amplitude of the carrier is $U_{t r}$, the normal modulation is:

$$
u_{\text {nom }}=U_{\text {nom }} \sin \left(\omega_{s} t\right)
$$

where $U_{\text {nom }}$ and $\omega_{s}$ are the amplitude and the angular frequency of the normal modulation, respectively.

The function of $u_{r 3}$ in the positive half cycle (denoted as $u_{r 3 p}$ ) has the same format as the normal one.

The function of $u_{r 1}$ in the positive half cycle is:

$$
u_{r 1 p}=U_{\text {nom }} \sin \left(\omega_{s} t\right)-2 U_{t r}
$$

The function of $u_{r 1}$ in the negative half cycle is:

$$
u_{r 1 n}=U_{t r}+U_{\text {nот }} \sin \left(\omega_{s} t\right)
$$

The function of $u_{r 2}$ in the positive half cycle is:

$$
u_{r 2 p}=U_{n o m} \sin \left(\omega_{s} t\right)-U_{t r}
$$

The function of $u_{r 2}$ in the negative half cycle is:

$$
u_{r 2 n}=2 U_{t r}+U_{n o m} \sin \left(\omega_{s} t\right)
$$

The function of $u_{r 3}$ in the negative half cycle is:

$$
u_{r 3 n}=3 U_{t r}+U_{n o m} \sin \left(\omega_{s} t\right)
$$

The amplitude modulation ratio of the proposed TRM-SPWM scheme is:

$$
M=U_{n o m} / 3 U_{t r}
$$

\section{SPECTRAL CHARACTERISTICS}

The phase angles of the modulations and the carrier are defined as:

$$
x=\omega_{c} t, y=\omega_{s} t
$$

where $\omega_{c}$ is the angular frequency of the carrier. Since the modulations and the carrier are all periodic, the output voltage $u_{\text {out }}$ can be defined as a double Fourier series:

$$
\begin{aligned}
u_{\text {out }}= & \frac{1}{2} A_{00}+\sum_{m=1}^{\infty}\left[A_{m 0} \cos (m x)+B_{m 0} \sin (m x)\right] \\
& +\sum_{m=1}^{\infty} \sum_{n=-\infty}^{\infty}\left[A_{m n} \cos (m x+n y)+B_{m n} \sin (m x+n y)\right]
\end{aligned}
$$




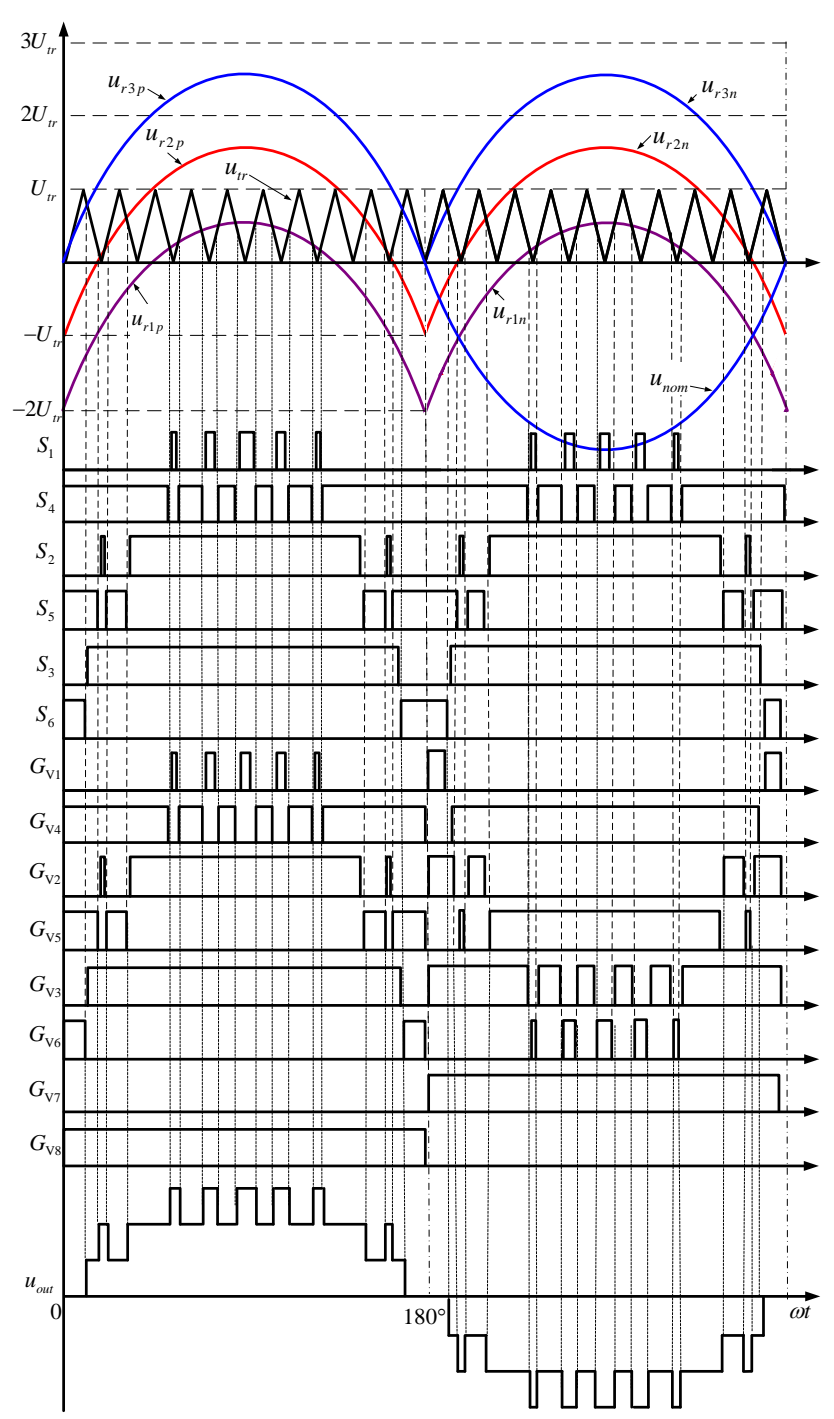

Fig. 2. Schematic diagram of conventional seven-level SPWM.

where:

$$
C_{m n}=A_{m n}+j B_{m n}=\frac{1}{2 \pi^{2}} \int_{0}^{2 \pi} \int_{-\pi}^{\pi} u_{\text {out }} e^{j(m x+n y)} d x d y
$$

The key point of calculating $C_{m n}$ is to determine the integral limitations of $x$ and $y$. The integral limitations of $y$ are determined first. Fig. 4 shows simplified schematic diagrams of the two strategies with $M>0.67$. It can be seen that during each cycle of the modulation, there exist eight "critical angles". At each critical angle, a modulation is equal to the amplitude of the carrier, and the level number of the output voltage changes. Within different regions (distinguished by the various critical angles), only one modulation is compared with the carrier. By calculating these critical angles, the corresponding functions of the modulations compared with the carrier in various regions can be determined.

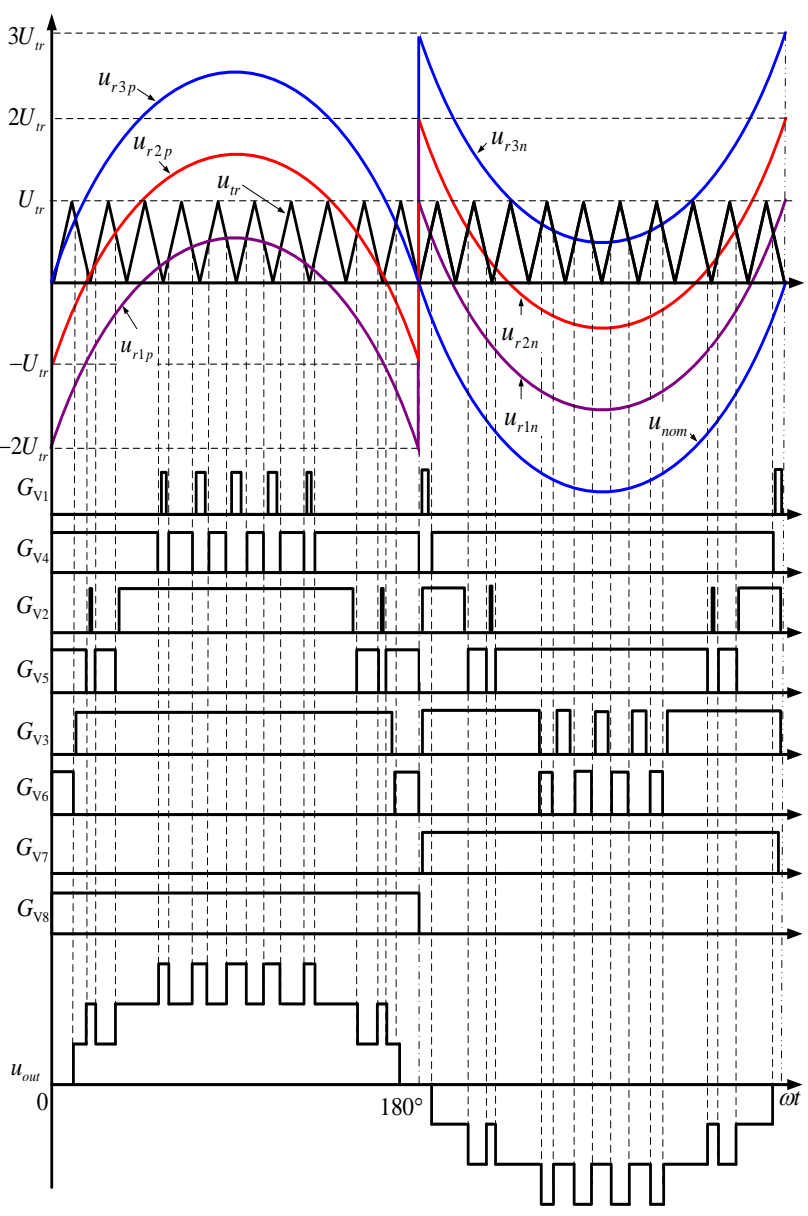

Fig. 3. Schematic diagram of proposed seven-level SPWM.

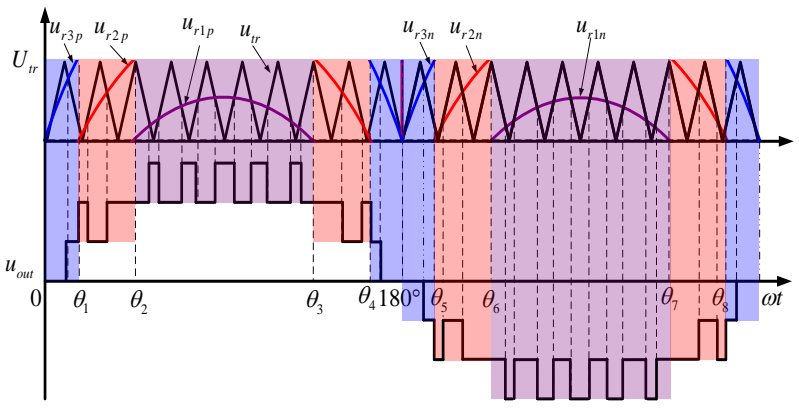

(a)

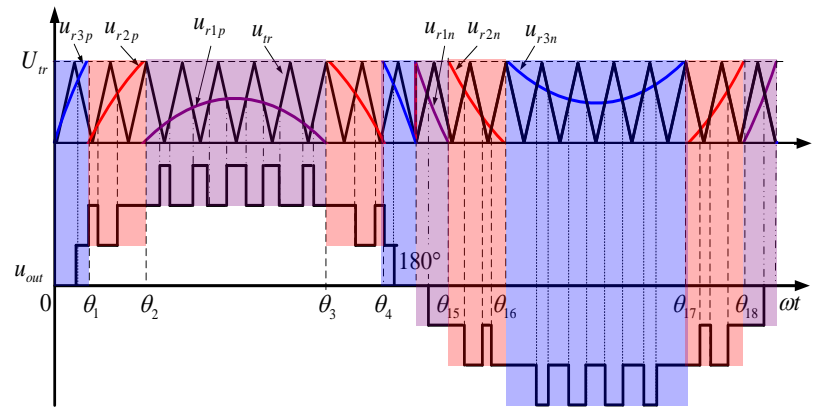

(b)

Fig. 4. Simplified schematic diagrams of the two SPWMs ((a) Conventional, (b) Proposed). 
The critical angles are first calculated with $0.67<M \leq 1$ as follows. For the conventional scheme, at every critical point, the following equations yield according to Fig. 4, equation (1), (3) and (5):

$$
\left\{\begin{array}{c}
U_{t r}=U_{\text {nom }} \sin \left(\theta_{1}\right), U_{t r}=U_{\text {nom }} \sin \left(\theta_{2}\right)-U_{t r} \\
\theta_{3}=\pi-\theta_{2}, \theta_{4}=\pi-\theta_{1} \\
U_{t r}=U_{n o m} \sin \left(\theta_{5}-\pi\right), U_{t r}=U_{n o m} \sin \left(\theta_{6}-\pi\right)-U_{t r} \\
\theta_{7}=3 \pi-\theta_{6}, \theta_{8}=3 \pi-\theta_{5}
\end{array}\right.
$$

Then the critical angles are calculated as:

$$
\left\{\begin{array}{c}
\theta_{1}=\sin ^{-1}\left(\frac{U_{t r}}{U_{\text {nom }}}\right), \theta_{2}=\sin ^{-1}\left(\frac{2 U_{t r}}{U_{\text {nom }}}\right) \\
\theta_{3}=\pi-\sin ^{-1}\left(\frac{2 U_{t r}}{U_{\text {nom }}}\right), \theta_{4}=\pi-\sin ^{-1}\left(\frac{U_{t r}}{U_{\text {nom }}}\right) \\
\theta_{5}=\pi+\sin ^{-1}\left(\frac{U_{t r}}{U_{\text {nom }}}\right), \theta_{6}=\pi+\sin ^{-1}\left(\frac{2 U_{t r}}{U_{\text {nom }}}\right) \\
\theta_{7}=2 \pi-\sin ^{-1}\left(\frac{2 U_{t r}}{U_{\text {nom }}}\right), \theta_{8}=2 \pi-\sin ^{-1}\left(\frac{U_{t r}}{U_{\text {nom }}}\right)
\end{array}\right.
$$

As for the proposed scheme, because it has the same modulation waveform as the conventional one in the positive half cycle, only the negative half cycle of the modulation needs be analyzed. The following equation yields according to Fig.4 (b):

$$
\left\{\begin{array}{c}
0=U_{\text {tr }}+U_{\text {nom }} \sin \left(\theta_{15}\right), 0=2 U_{\text {tr }}+U_{\text {nom }} \sin \left(\theta_{16}\right) \\
\theta_{17}=3 \pi-\theta_{16}, \theta_{18}=3 \pi-\theta_{15}
\end{array}\right.
$$

Then the critical angles in the negative half cycle of modulation are calculated as:

$$
\left\{\begin{array}{c}
\theta_{15}=\pi+\sin ^{-1}\left(\frac{U_{t r}}{U_{\text {nom }}}\right)=\theta_{5}, \theta_{16}=\pi+\sin ^{-1}\left(\frac{2 U_{t r}}{U_{\text {nom }}}\right)=\theta_{6} \\
\theta_{17}=2 \pi-\sin ^{-1}\left(\frac{2 U_{t r}}{U_{\text {nom }}}\right)=\theta_{7}, \theta_{18}=2 \pi-\sin ^{-1}\left(\frac{U_{t r}}{U_{\text {nom }}}\right)=\theta_{8}
\end{array}\right.
$$

When $0.33<M \leq 0.67, u_{r 1 p}$ is always smaller than zero, and $u_{r 3 n}$ is always larger than $U_{t r}$, and the critical angles are determined as:

$$
\theta_{1}=\sin ^{-1}\left(\frac{U_{t r}}{U_{\text {nот }}}\right), \theta_{2}=\theta_{3}=\frac{\pi}{2}, \theta_{6}=\theta_{7}=\frac{3 \pi}{2}, \theta_{8}=2 \pi-\theta_{1}
$$

When $M \leq 0.33$, only $u_{r 3 p}$ and $u_{r 1 n}$ are compared with the carrier, and the critical angles determined as:

$$
\theta_{1}=\theta_{2}=\theta_{3}=\theta_{4}=\frac{\pi}{2}, \theta_{5}=\theta_{6}=\theta_{7}=\theta_{8}=\frac{3 \pi}{2}
$$

In the following, the integral limitations of $x$ with respect to different values of the output voltage level are analyzed. According to the relationship between the various modulations and the carrier, the ranges of the phase angle within one carrier cycle corresponding to a different number of the output voltages level of the two strategies are derived. The results are presented in TABLE I and TABLE II.
TABLE I

The INTEgral Limitations Of $X$ AND $Y$ Of ConventionaL SCHEME

\begin{tabular}{|ccc|}
\hline$u_{\text {out }}(\mathrm{pu})$ & $y$ & $x$ \\
1 & $0 \leq y<\theta_{1}$ & $-3 \pi M \sin y \leq x \leq 3 \pi M \sin y$ \\
2 & $\theta_{1} \leq y<\theta_{2}$ & $\pi-3 \pi M \sin y \leq x \leq-\pi+3 \pi M \sin y$ \\
3 & $\theta_{2} \leq y<\theta_{3}$ & $2 \pi-3 \pi M \sin y \leq x \leq-2 \pi+3 \pi M \sin y$ \\
2 & $\theta_{3} \leq y<\theta_{4}$ & $\pi-3 \pi M \sin y \leq x \leq-\pi+3 \pi M \sin y$ \\
1 & $\theta_{4} \leq y<\pi$ & $-3 \pi M \sin y \leq x \leq 3 \pi M \sin y$ \\
-1 & $\pi \leq y<\theta_{5}$ & $3 \pi M \sin y \leq x \leq-3 \pi M \sin y$ \\
-2 & $\theta_{5} \leq y<\theta_{6}$ & $\pi+3 \pi M \sin y \leq x \leq-\pi-3 \pi M \sin y$ \\
-3 & $\theta_{6} \leq y<\theta_{7}$ & $2 \pi+3 \pi M \sin y \leq x \leq-2 \pi-3 \pi M \sin y$ \\
-2 & $\theta_{7} \leq y<\theta_{8}$ & $\pi+3 \pi M \sin y \leq x \leq-\pi-3 \pi M \sin y$ \\
-1 & $\theta_{8} \leq y<2 \pi$ & $3 \pi M \sin y \leq x \leq-3 \pi M \sin y$ \\
\hline
\end{tabular}

TABLE II

The InTEgRal Limitations Of $X$ And $Y$ Of Proposed Scheme

$\begin{array}{ccc}u_{\text {out }}(\mathrm{pu}) & y & x \\ 1 & 0 \leq y<\theta_{1} & -3 \pi M \sin y \leq x \leq 3 \pi M \sin y \\ 2 & \theta_{1} \leq y<\theta_{2} & \pi-3 \pi M \sin y \leq x \leq-\pi+3 \pi M \sin y \\ 3 & \theta_{2} \leq y<\theta_{3} & 2 \pi-3 \pi M \sin y \leq x \leq-2 \pi+3 \pi M \sin y \\ 2 & \theta_{3} \leq y<\theta_{4} & \pi-3 \pi M \sin y \leq x \leq-\pi+3 \pi M \sin y \\ 1 & \theta_{4} \leq y<\pi & -3 \pi M \sin y \leq x \leq 3 \pi M \sin y \\ -1 & \pi \leq y<\theta_{5} & \pi+3 \pi M \sin y \leq x \leq \pi-3 \pi M \sin y \\ -2 & \theta_{5} \leq y<\theta_{6} & 2 \pi+3 \pi M \sin y \leq x \leq-3 \pi M \sin y \\ -3 & \theta_{6} \leq y<\theta_{7} & 3 \pi+3 \pi M \sin y \leq x \leq-\pi-3 \pi M \sin y \\ -2 & \theta_{7} \leq y<\theta_{8} & 2 \pi+3 \pi M \sin y \leq x \leq-3 \pi M \sin y \\ -1 & \theta_{8} \leq y<2 \pi & \pi+3 \pi M \sin y \leq x \leq \pi-3 \pi M \sin y\end{array}$

Then the contour curves are plotted in Fig. 5. Each curve defines a transition in the voltage. It can be seen that the contours curves of the two strategies are different, which means different Fourier integral results. Utilizing the superposition principle, the Fourier integrals of the conventional scheme yield from TABLE I as:

$$
\begin{aligned}
& {\left[\begin{array}{l}
\int_{0}^{\theta_{1}} \int_{-3 \pi M \sin y}^{3 \pi M \sin y} e^{j(m x+n y)} d x d y+\int_{\theta_{1}}^{\theta_{2}-\pi+3 \pi M \sin y} \int_{\pi-3 \pi \sin y}^{\theta_{3}} e^{j(m x+n y)} d x d y \\
+\int_{\theta_{2}}^{\theta_{2}-2 \pi+3 \pi M \sin y} \int_{2 \pi-3 \pi M \sin y}^{\theta_{4}-\pi+3 \pi M \sin y} e^{j(m x+n y)} d x d y+\int_{\theta_{3}}^{j(m-3 \pi M \sin y} e^{j(m x+n y)} d x d y
\end{array}\right.} \\
& C_{m n}=\frac{1}{2 \pi^{2}}+\int_{\theta_{4}-3 \pi M \sin y}^{\pi} \int^{3 \pi M \sin y} e^{j(m x+n y)} d x d y-\int_{\pi}^{\theta_{5}-3 \pi M \sin y} \int_{3 \pi M \sin y}^{j(m x+n y)} d x d y \\
& -\int_{\theta_{5}}^{\theta_{6}-\pi-3 \pi M \sin y} \int_{\pi=\sin y}^{j(m x+n y)} d x d y-\int_{\theta_{6}}^{\theta_{7}-2 \pi-3 \pi M \sin y} \int_{2 \pi / 3 \sin y}^{3} e^{j(m x+n y)} d x d y \\
& -\int_{\theta_{7}}^{\theta_{8}-\pi-3 \pi M \sin y} \int_{\pi=\sin y}^{j(m x+n y)} d x d y-\int_{\theta_{8}}^{2 \pi-3 \pi M \sin y} \int_{3 \pi \sin y}^{j(m x+n y)} d x d y
\end{aligned}
$$




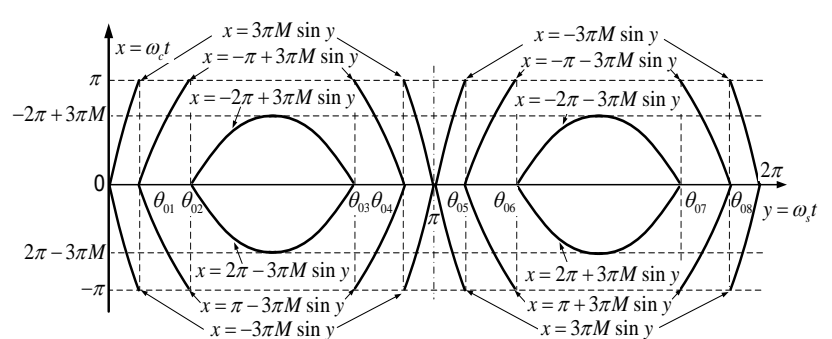

(a)

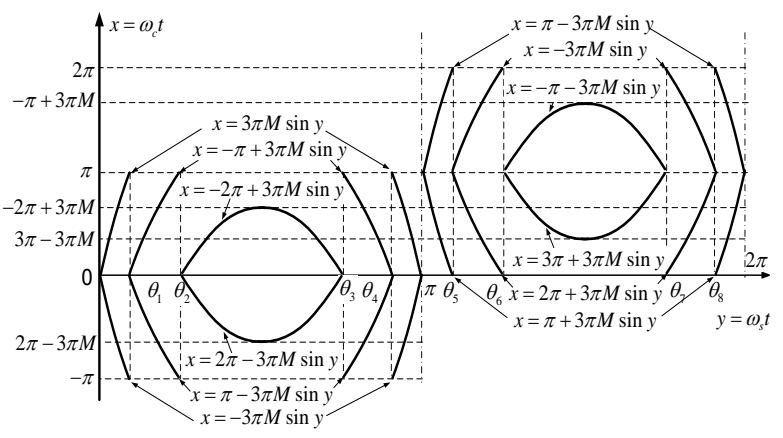

(b)

Fig. 5. Contour curves within $2 \pi$ phase wraparound ((a) Conventional, (b) Proposed).

The Fourier integrals of the proposed scheme yield from TABLE II as:

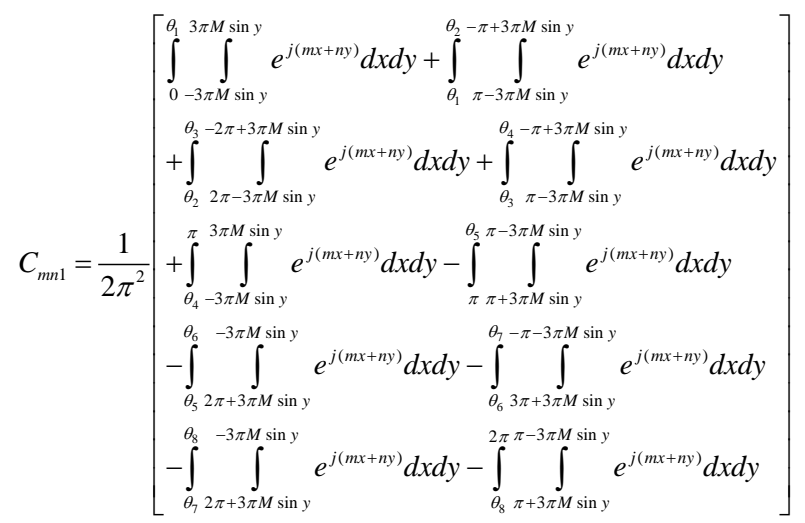

The expansion formula of the Jacobi-angle series is:

$$
\sin (\xi \sin y)=2 \sum_{k=1, \text { odd }}^{\infty}\left[J_{k}(\xi) \sin (k y)\right]
$$

Setting $\xi=3 m M \pi$, the Fourier series of the output voltage of the conventional scheme yields:

$$
u_{\text {out }}=M \sin \left(\omega_{s} t\right)+\frac{2}{3 \pi^{2}} \sum_{m=1}^{\infty} \frac{1}{m} \sum_{n=-\infty}^{\infty}\left[B_{m n} \sin \left(m \omega_{c} t+n \omega_{s} t\right)\right]
$$

where:

$$
\begin{aligned}
B_{m n}= & J_{n}(3 m M \pi)\left[1-(-1)^{n}\right]\left\{\sum_{k=1}^{3}\left\{\cos [m(k-1) \pi]\left(\theta_{k+1}-\theta_{k}\right)\right\}\right\} \\
& -\sum_{h \neq-n, \text { odd }}^{\infty}\left\{\times \sum_{k=1}^{3}\left\{\cos [m(k-1) \pi]\left\{\begin{array}{l}
\sin \left[(n+h) \theta_{k+1}\right] \\
-\sin \left[(n+h) \theta_{k}\right]
\end{array}\right\}\right\}\right\}
\end{aligned}
$$

The Fourier series of the output voltage of the proposed scheme is:

$$
u_{\text {out }}=M \sin \left(\omega_{s} t\right)+\frac{2}{3 \pi^{2}} \sum_{m=1}^{\infty} \frac{1}{m} \sum_{n=-\infty}^{\infty}\left[\begin{array}{l}
A_{m n 1} \cos \left(m \omega_{c} t+n \omega_{s} t\right) \\
+B_{m n 1} \sin \left(m \omega_{c} t+n \omega_{s} t\right)
\end{array}\right]
$$

where:

$$
\begin{aligned}
A_{m n 1}= & {\left[1-(-1)^{m}\right]\left[1+(-1)^{n}\right] } \\
\times & \sum_{h \neq-n, \text { odd }}^{\infty}\left\{\begin{array}{l}
J_{h}(3 m M \pi) \frac{1}{n+h} \\
\times \sum_{k=1}^{3}\left\{\cos [m(k-1) \pi]\left\{\cos \left[(n+h) \theta_{k+1}\right]-\cos \left[(n+h) \theta_{k}\right]\right\}\right\}
\end{array}\right\} \\
B_{m n 1}= & {\left[1+(-1)^{m}\right]\left[1-(-1)^{n}\right] } \\
& \times\left\{\begin{array}{l}
J_{n}(3 m M \pi) \sum_{k=1}^{3}\left\{\cos [m(k-1) \pi]\left(\theta_{k+1}-\theta_{k}\right)\right\} \\
\left.-\sum_{h \neq-n, \text { odd }}^{\infty}\left\{\times \sum_{k=1}^{3}\left\{\cos [m(k-1) \pi]\left\{\begin{array}{l}
\sin \left[(n+h) \theta_{k+1}\right] \\
-\sin \left[(n+h) \theta_{k}\right]
\end{array}\right\}\right\}\right)\right\}
\end{array}\right.
\end{aligned}
$$

It can be seen from equations (21) and (23) that the spectral characteristics of the two strategies are different. There are no harmonics at the carrier frequency and its multiples in the conventional scheme. No sideband harmonics exist when $m$ and $n$ are both odd or are both even at the same time in proposed scheme.

\section{Simulation Results}

The proposed TRM-SPWM is simulated with MATLAB/SIMULINK software. The simulation model parameters are as follows: the total DC voltage $E$ is $45 \mathrm{~V}$, the frequency of the carrier is $10 \mathrm{kHz}$, the frequency of the modulation wave is $50 \mathrm{~Hz}$, and $M=0.8$. The corresponding simulation waveforms are shown in Fig. 6. It can be seen that the uniform modulation waveforms and the PWM waveforms from Fig. 2 are obtained. The total output voltage is seven-level, and its low pass filtering waveform $\left(u_{L P F}\right)$ is better than sinusoidal.

Since the differences between the total harmonics disturbances (THDs) of the two strategies are not distinct from the theoretical results, the spectral characteristics of the two strategies are compared in the simulation. Firstly, the spectrum with a lower frequency ratio $(p=20)$ and $M=0.8$ are simulated, and the results are shown in Fig. 7. The distributions of the spectrum of the two strategies are different, but the THDs are almost the same.

Furthermore, the THDs, the fundamental components of the output voltage ( $U_{\text {out }}$ ) and the differences between the two SPWMs with different amplitude modulation ratios and frequency ratios are simulated. The distribution curves are shown in Fig. 8. $\Delta U_{\text {out }}$ and $\Delta$ THD denote the differences between the output voltages and the THDs. It can be seen from the figures that when the frequency ratio is higher, the differences between the THDs of the two strategies have become so tiny that they can almost be ignored. 

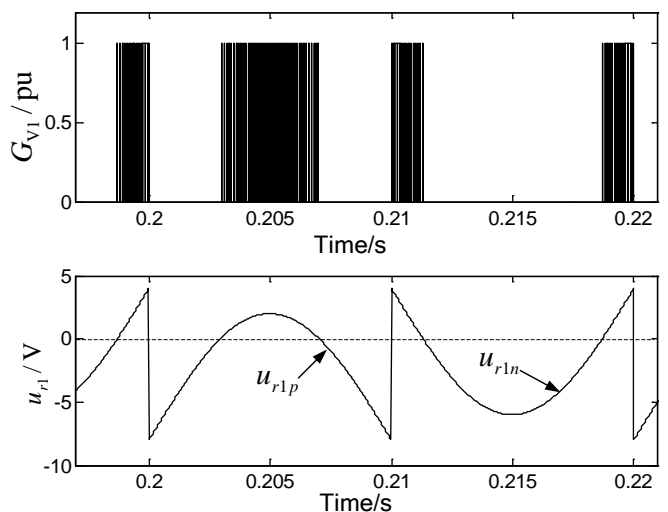

(a)
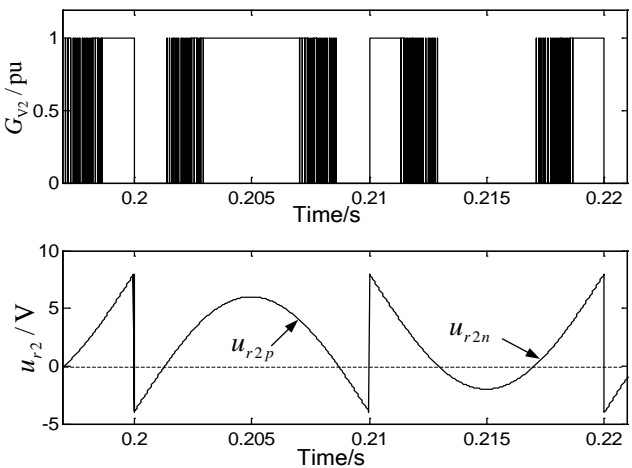

(b)
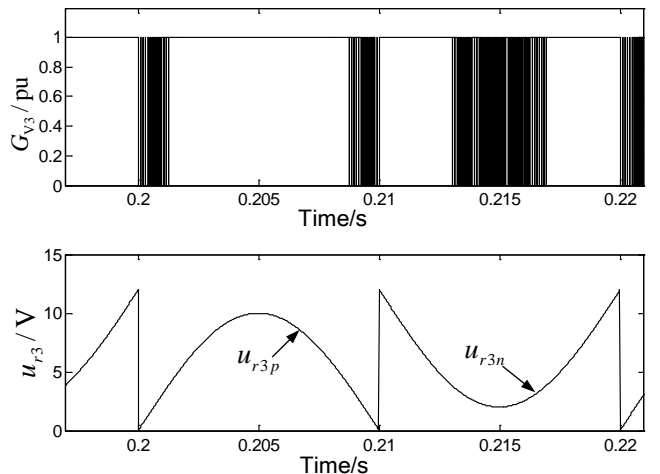

(c)
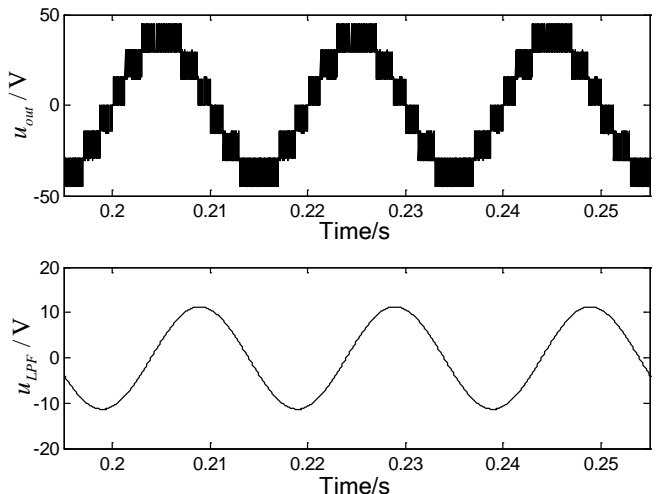

(d)

Fig. 6. Simulation waveforms of proposed seven-level SPWM ((a) $G_{\mathrm{V} 1}$ and $u_{r 1}$, (b) $G_{\mathrm{V} 2}$ and $u_{r 2}$, (c) $G_{\mathrm{V} 3}$ and $u_{r 3}$, (d) $u_{\text {out }}$ and $u_{L P F}$ ).

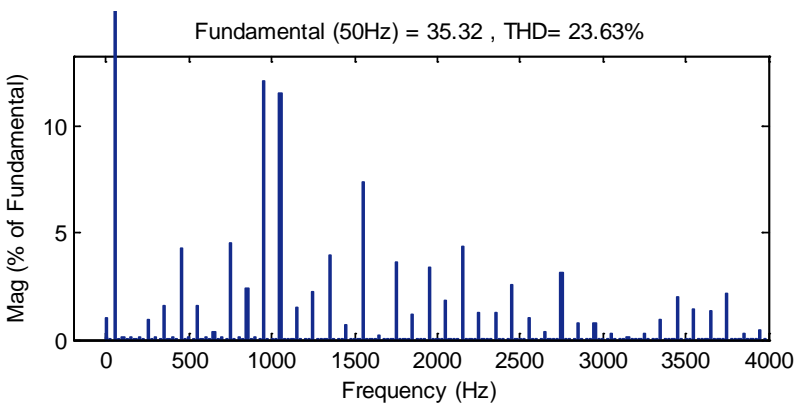

(a)

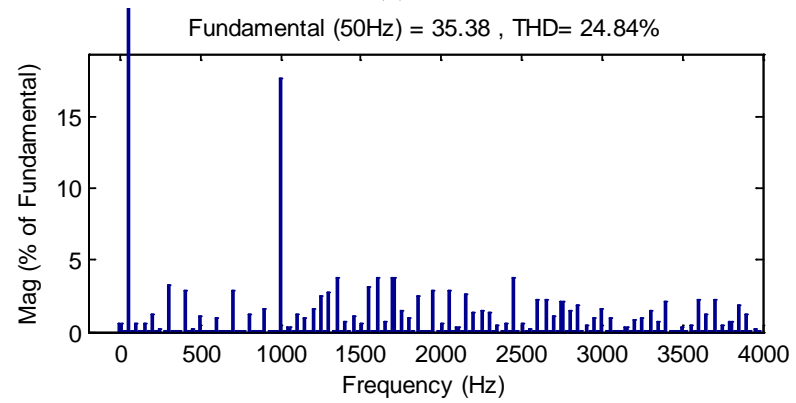

(b)

Fig. 7. FFTs of output voltages with $M=0.8$ and $p=20$ ((a) Conventioanl, (b) Proposed).

\section{IMPLEMENTATION AND EXPERIMENTAL RESULTS}

An experimental prototype of a seven-level inverter with an RC low-pass filter and a DSP chip (TMS30F2812) has been set up. The experimental parameters are the same as the ones from the simulation model. The timer T1 in the DSP is used to generate the carrier. To obtain six PWM signals, all three of the compare registers in the DSP chip are used. The IO ports GPIOA6 and GPIOA7 are used to output the control signals of $\mathrm{V} 7$ and $\mathrm{V} 8$.

The experimental results with $M=0.8$ are shown in Fig.9. From Fig.9, it can be seen that the actual three modulations have the same shapes as Fig.2 and Fig.3. The output voltage waveform $u_{\text {out }}$ is seven-level, and its low-pass waveform $u_{L P F}$ is better than sinusoidal. The experimental results with $M=0.3$ and 0.5 are presented as well. They correspond to the cases of the three-level and five-level output voltage waveforms. As shown in Fig. 10, the output voltages with different amplitude ratios are all implemented correctly. The above experimental results illustrate that the correct output voltage PWM waveforms within the whole range of the amplitude modulation ratio are all obtained with the proposed scheme. Thus the single-phase seven-level asymmetry diodeclamped inverter is driven correctly with the simple implementation scheme of a multilevel SPWM. 


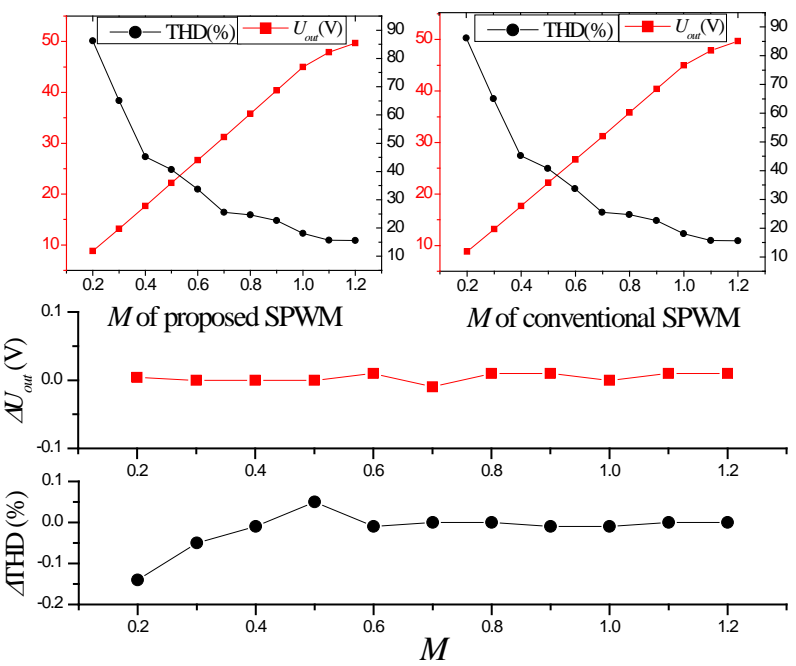

(a)

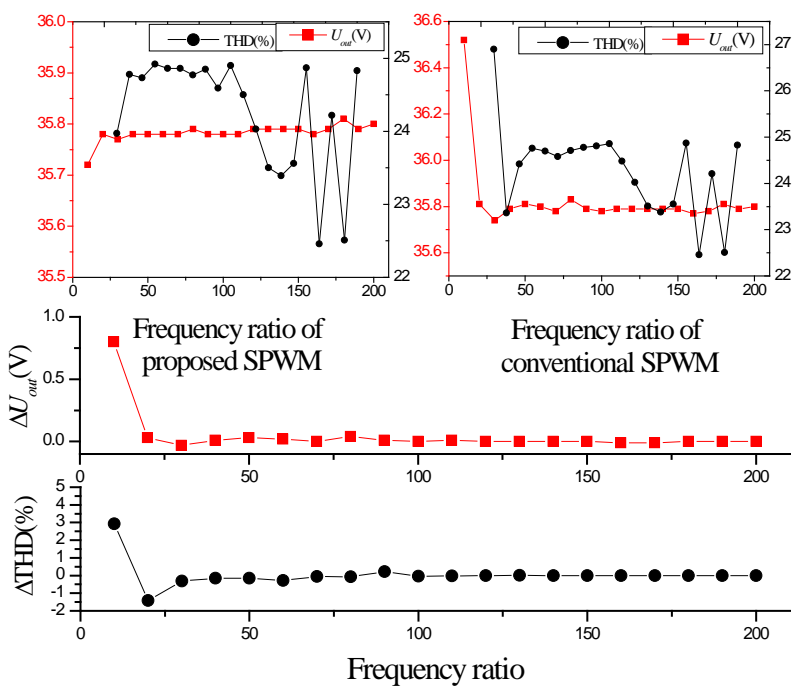

(b)

Fig. 8. THD curves of output voltage versus $M$ and frequency ratio of conventional and proposed PWM strategies ((a) Different $M$ s with $p=199$, (b) Different frequency ratios and $M=0.8$ ).

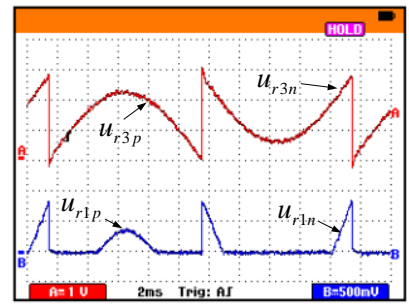

(a)

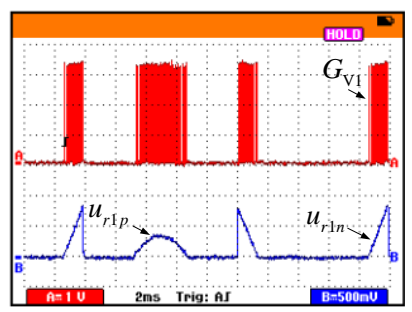

(c)

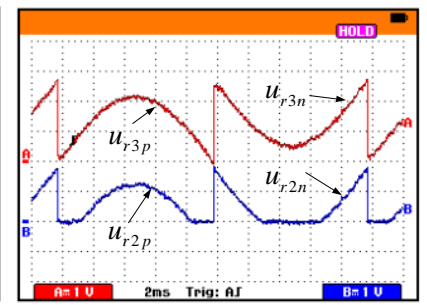

(b)

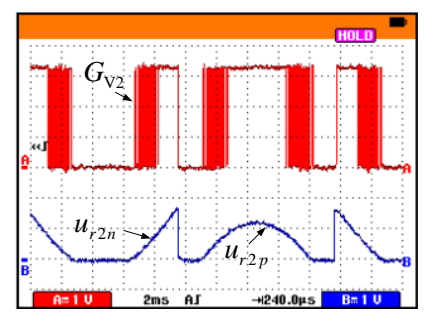

(d)

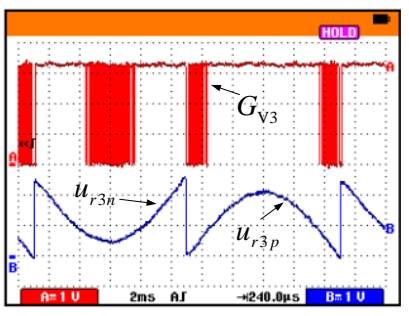

(e)

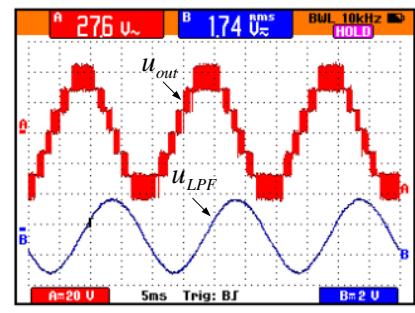

(f)
Fig. 9. Experimental waveforms of proposed seven-level SPWM (M=0.8). ((a) $u_{r 1}$ and $u_{r 2}$, (b), $u_{r 1}$ and $u_{r 3}$, (c) $G_{\mathrm{V} 1}$ and $u_{r 1}$, (d) $G_{\mathrm{V} 2}$ and $u_{r 2}$, (e) $G_{\mathrm{V} 3}$ and $u_{r 3}$, (f) $u_{\text {out }}$ and $u_{L P F}$ ).

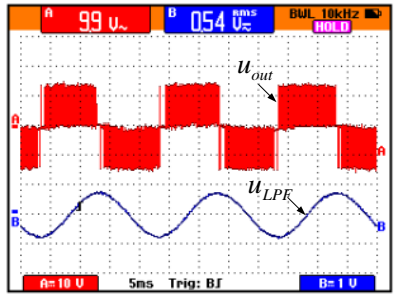

(a)

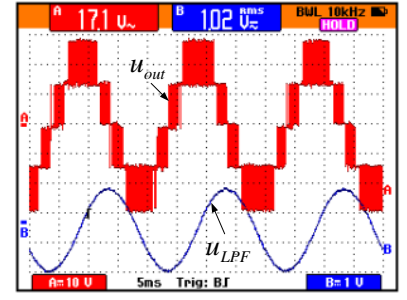

(b)
Fig. 10. Experimental waveforms of output voltage and its low-pass filter under different amplitude modulation ratios ((a) $M=0.3$, (b) $M=0.5$ ).

\section{CONCLUSIONS}

The proposed TRM-SPWM scheme is implemented with only one DSP chip. The implementation platform is simplified, the cost is reduced and the reliability is enhanced. The experimental results verify its accuracy and feasibility. The TRM-SPWM has spectral characteristics that are similar to those of the conventional scheme. This indicates that the proposed scheme can entirely replace the conventional one to control a single-phase seven-level asymmetry diode-clamped inverter. This scheme has excellent practicality.

\section{ACKNOWLEDGMENT}

This work was supported by the National Natural Science Foundation of China (Grant No. 51107018), the Research Fund for the Doctoral Program of Higher Education of China (Grant No. 20102302120013) and Fundamental Research Funds for the Central Universities of China (Grant No. HIT. NSRIF. 2010103).

\section{REFERENCES}

[1] J. Rodriguez, S. Bernet, B. Wu, J. O. Pontt, and S. Kouro, "Multilevel voltage-source-converter topologies for industrial medium-voltage drives," IEEE Trans. Ind. Electron., Vol. 54, No. 6, pp. 2930-2945, Dec. 2007.

[2] C. Cecati, F. Ciancetta, and P. Siano, "A multilevel inverter for photovoltaic systems with fuzzy logic control," IEEE Trans. Ind. Electron., Vol. 57, No. 12, pp. 4115-4125, Dec. 2010.

[3] E. Villanueva, P. Correa, J. Rodríguez, and M. Pacas, "Control of a single phase cascaded H-bridge multilevel inverter for grid-connected photovoltaic systems," IEEE 
Trans. Ind. Electron., Vol. 56, No. 11, pp. 4399-4406, Nov. 2009.

[4] N. A. Rahim and J. Selvaraj, "Multi-string five-level inverter with novel PWM control scheme for PV application,” IEEE Trans. Ind. Electron., Vol. 57, No. 6, pp. 2111-2121, Jun. 2010.

[5] M. M. Renge and H. M. Suryawanshi, "Five-level diode clamped inverter to eliminate common mode voltage and reduce $\mathrm{dv} / \mathrm{dt}$ in medium voltage rating induction motor drives,” IEEE Trans. Power Electron., Vol. 23, No. 4, pp. 1598-1160, Jul. 2008.

[6] J. Huang and K. A. Corzine, "Extended operation of flying capacitor multilevel inverter,” IEEE Trans. Power Electron., Vol. 21, No. 1, pp. 140-147, Jan. 2006.

[7] T. A. Meynard, "Multicell converters: basic concepts and industry applications,” IEEE Trans. Ind. Electron., Vol. 49, No. 5, pp. 955-964, Oct. 2002.

[8] S. Kouro, J. Rebolledo, and J. Rodriguez, "Reduced switching-frequency-modulation algorithm for high-power multilevel inverters,” IEEE Trans. Ind. Electron., Vol. 54, No. 5, pp. 2894-2901, Sep. 2007.

[9] L. M. Tolbert and T. G. Habetler, "Novel multilevel inverter carrier-based PWM method," IEEE Trans. Ind. Appl., Vol. 35, No. 5, pp. 1098-1106, May 1999.

[10] H. M. Kojabadi, B. Yu, I. A. Gadoura, L. Chang, and M. Ghribi, "A novel DSP-based current-controlled PWM strategy for single phase grid connected inverters," IEEE Trans. Power Electronics, Vol. 21, No. 4, pp. 985-993, Jul. 2006.

[11] Z. C. Liu, P. J. Kong, X. Z. Wu, and L. P. Huang, "Implementation of dsp-based three-level inverter with dead time compensation”, IEEE Int. Power Electronics and Motion Control Conf., pp. 782-787, 2004.

[12] S. M. Li and L.Y Xu, “A dsp peripheral design for three-level inverter space vector pwm modulations," IEEE Int. Power Electronics Specialist Conf., pp. 189-194, 2003.

[13] L. H. Barreto, P. Pracap, C. M. T. Cruz, and R. T. Bascope, "PID digital control using microcontroller and FPGA applied to a single-phase three-level inverter," IEEE Int. Applied Power Electronics Conf., pp. 1443-1446, 2007.

[14] C. Govindaraju and K. Baskaran, "Analysis and implementation of multiphase multilevel hybrid single carrier sinusoidal modulation," Journal of Power Electronics, Vol. 10, No. 4, pp. 365-373, Jul. 2010.

[15] A. R. Nasrudin, C. Krismadinata and S. Jeyraj, "Single-phase seven-level grid-connected inverter for photovoltaic system,” IEEE Trans. Ind. Electron., Vol. 58, No. 6, pp. 2435-2443, Jun. 2011.

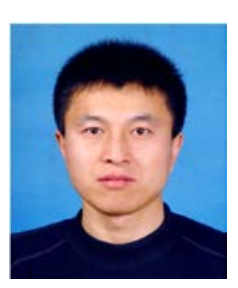

Fengjiang Wu was born in Harbin, China. He received his B.S., M.S. and Ph.D. in Electrical Engineering from the Harbin Institute of Technology, Harbin, China, in 2002, 2004 and 2007, respectively. Since 2007, he has been with the Department of Electrical Engineering, Harbin Institute of Technology, where he is currently a Lecturer of Electrical Machine and Apparatus. His current research interests include renewable energy generation, multilevel inverter technologies and electric machine drives.

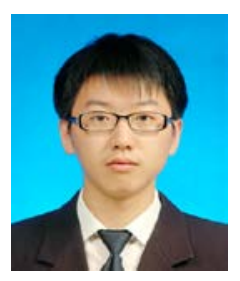

Bo Sun was born in Heilongjiang Province, China. He received his B.S. in Electrical Engineering from the Harbin Institute of Technology, Harbin, China, in 2011. He is currently pursuing his M.S. from the Department of Electrical Engineering, Harbin Institute of Technology. His current research interests include grid-connected inverters for renewable energy generation.

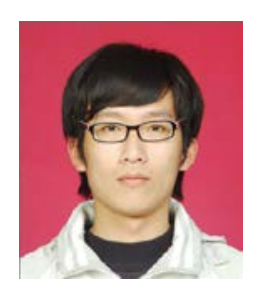

Lujie Zhang was born in Fujian Province, China. He received his B.S. in Electrical Engineering from the Harbin Institute of Technology, Harbin, China, in 2012. He is currently pursuing his M.S. from the Department of Electrical Engineering, Harbin Institute of Technology. His current research interest include grid-connected inverters for renewable energy generation.

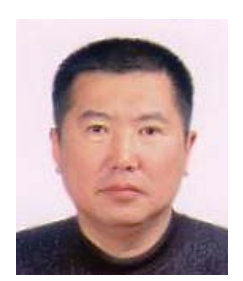

Li Sun was born in Heilongjiang Province, China. He received his B.S., M.S. and Ph.D. in Electrical Engineering from the Harbin Institute of Technology, Harbin, China, in 1982, 1986, and 1991, respectively. Since 1986, he has been with the Department of Electrical Engineering, Harbin Institute of Technology, where he is currently a Professor and Doctoral Supervisor of Electrical Machines and Apparatus. His current research interests include electric machines and drives, power electronics and applications, and electromagnetic compatibility. 